\title{
Antenatal Corticosteroids for Women at Risk of Late Preterm Delivery
}

\author{
Cynthia Gyamfi-Bannerman, MD, MSc, Elizabeth A. Thom, PhD, Sean C. Blackwell, MD, \\ Alan T.N. Tita, MD, PhD, Uma M. Reddy, MD, MPH, George R. Saade, MD, Dwight J. Rouse, \\ MD, David S. McKenna, MD, Erin A.S. Clark, MD, John M. Thorp Jr., MD, Edward K. Chien, \\ MD, MBA, Alan M. Peaceman, MD, Ronald S. Gibbs, MD, Geeta K. Swamy, MD, Mary E. \\ Norton, MD, Brian M. Casey, MD, Steve N. Caritis, MD, Jorge E. Tolosa, MD, MSCE, Yoram \\ Sorokin, MD, J. Peter VanDorsten, MD, and Lucky Jain, MD, MBA \\ Columbia University, New York (C.G-B.); the George Washington University Biostatistics Center, \\ Washington, DC (E.A.T.); the University of Texas Health Science Center at Houston-Children's \\ Memorial Hermann Hospital, Houston (S.C.B.); University of Alabama at Birmingham, \\ Birmingham (A.T.N.T.); the Eunice Kennedy Shriver National Institute of Child Health and Human \\ Development, Bethesda, MD (U.M.R.); University of Texas Medical Branch, Galveston (G.R.S.); \\ Brown University, Providence, RI (D.J.R.); The Ohio State University, Columbus (D.S.M.); \\ University of Utah Health Sciences Center, Salt Lake City (E.A.S.C.); University of North Carolina \\ at Chapel Hill, Chapel Hill (J.M.T.); Northwestern University, Chicago (A.M.P.); MetroHealth \\ Medical Center-Case Western Reserve University, Cleveland (E.K.C.); University of Colorado \\ School of Medicine, Anschutz Medical Campus, Aurora (R.S.G.); Duke University, Durham, NC \\ (G.S.); Stanford University, Stanford, CA (M.E.N.); University of Texas Southwestern, Dallas \\ (B.M.C.); University of Pittsburgh, Pittsburgh (S.N.C.); Oregon Health \& Sciences University, \\ Portland (J.E.T.); Wayne State University, Detroit (Y.S.); Medical University of South Carolina, \\ Charleston, (J.P.V.D.); and Emory University, Atlanta, GA (L.J.)*
}

the Eunice Kennedy Shriver National Institute of Child Health and Human Development Maternal-Fetal Medicine Units Network

\begin{abstract}
Background-Infants born at 34 to 36 weeks' gestation (late preterm) have greater risks of adverse respiratory and other outcomes, than those born at 37 weeks gestation or later. It is not known whether betamethasone administered to women at risk for late preterm delivery decreases risks of neonatal morbidities.
\end{abstract}

Corresponding Author: Cynthia Gyamfi-Bannerman, MD, MSc, Associate Professor, Columbia University Medical Center, Department of Obstetrics and Gynecology, Division of Maternal-Fetal Medicine, 622 West $168^{\text {th }}$ Street, PH-16, New York, NY 10032.cg2231@cumc.columbia.edu..

* Additional Members of this network are listed in the Supplementary Appendix.

The comments and views expressed in this article are those of the authors and do not necessarily represent the views of the Eunice Kennedy Shriver National Institute of Child Health and Human Development (NICHD) or the National Heart Lung and Blood Institute (NHLBI). 
Methods-We conducted a multicenter randomized trial of women with a singleton gestation at high risk for late preterm delivery. Participants were randomized to two injections of $12 \mathrm{mg}$ betamethasone or matching placebo 24 hours apart. The primary outcome was a neonatal composite of treatment in the first 72 hours (continuous positive airway pressure or high flow nasal cannula for at least two hours, supplemental oxygen with a fraction of inspired oxygen of at least 30 percent for at least four hours, extra corporeal membrane oxygenation or mechanical ventilation) or stillbirth or neonatal death before 72 hours.

Results-2,831 patients were randomized. The primary outcome occurred in $11.6 \%$ of the betamethasone group versus $14.4 \%$, in the placebo group (Relative Risk 0.80, 95\% confidence interval 0.66-0.97, $\mathrm{P}=0.02$ ). Severe respiratory morbidity, transient tachypnea of the newborn, surfactant use, and bronchopulmonary dysplasia were also significantly less common in the betamethasone group. There were no significant differences between groups in the incidence of chorioamnionitis or neonatal sepsis. Neonatal hypoglycemia was more common in the betamethasone group. (24.0\% versus $14.9 \%$, RR 1.61, 95\% CI 1.38-1.88, $\mathrm{P}<0.001$ )

Conclusions-Administration of betamethasone to women at risk for late preterm delivery significantly reduced the rate of neonatal respiratory morbidity.

Antenatal corticosteroids are widely used in obstetrics for pregnancies at risk for early preterm delivery. Their use increased especially after a consensus conference held by the National Institutes of Health in 1994, which concluded that there was strong evidence that corticosteroids reduce adverse neonatal outcomes, including death, respiratory distress syndrome, and other morbidities when administered to women likely to deliver before 34 weeks. ${ }^{1-3}$ The recommendation was not extended to women at risk for preterm delivery after 34 weeks. This was both due to a lack of data ${ }^{4,5}$ and because it was believed that at a threshold of 34-35 weeks nearly all infants thrive, with survival at this gestational age being within $1 \%$ of survival at term. ${ }^{6}$ However, it is now clear that infants born during the 'late' preterm period ( 34 weeks 0 days to 36 weeks 6 days) have increased neonatal and childhood complications compared with newborns born at term (37 weeks or later). ${ }^{7-9}$ In that context, a workshop in 2005 recommended redirecting research to infants born between 34 and 36 weeks gestation, particularly to answer the question of whether antenatal corticosteroids are beneficial in this population. ${ }^{10}$ Currently $8 \%$ of all deliveries occur in the late preterm period; ${ }^{11}$ thus the potential public health and economic impact of decreasing respiratory and other morbidities associated with prematurity by administration of antenatal corticosteroids is considerable. We therefore designed a randomized trial to assess whether administration of betamethasone to women likely to deliver in the late preterm period decreases respiratory and other neonatal morbidities.

\section{Methods}

We conducted the trial at 17 university-based clinical centers participating in the NICHD Maternal-Fetal Medicine Units (MFMU) Network. The protocol was approved by the institutional review board at each center. Written informed consent was obtained from all participants before randomization. The protocol is available at NEJM.org. Drs. GyamfiBannerman, Thom and Reddy take responsibility for the accuracy and completeness of reporting and the fidelity of the report to the study protocol. 


\section{Screening and recruitment}

Women with a live singleton pregnancy at 34 weeks 0 days to 36 weeks 5 days of gestation and a high probability of delivery in the late preterm period were eligible for enrollment. High probability of delivery was defined as either 1) preterm labor with intact membranes, and at least $3 \mathrm{~cm}$ dilation or $75 \%$ cervical effacement, or 2) spontaneous rupture of the membranes, or 3) if neither of these criteria applied, expected preterm delivery for any other indication via induction or cesarean between 24 hours to 7 days after the planned randomization, as determined by the obstetric provider. A woman was ineligible if she was expected to deliver in less than 12 hours for any reason including: 1) ruptured membranes in the presence of more than 6 contractions per hour or cervical dilation of 3 centimeters or more unless oxytocin was withheld for at least 12 hours (although other induction agents were allowed), 2) chorioamnionitis, 3) cervical dilation of $8 \mathrm{~cm}$ or more, and 4) evidence of non-reassuring fetal status requiring immediate delivery. A woman was also excluded if she had previously received antenatal corticosteroids for fetal lung maturation during this pregnancy, if she was a candidate for stress dose corticosteroids, if she had a contraindication to betamethasone, pre-gestational diabetes, or a known major fetal anomaly. Gestational age was determined by a standardized method across sites; if a dating ultrasound had not been performed before 32 weeks for a woman with known date of last menstrual period or before 24 weeks for those with unknown date of last menstrual period, she was excluded. Full eligibility criteria are provided in the Supplementary Appendix.

\section{Study Protocol}

Eligible and consenting women were randomly allocated in a 1:1 ratio to a course of two intramuscular injections containing either $12 \mathrm{mg}$ of betamethasone or matching placebo, 24 hours apart. The randomization sequence was prepared by the independent data coordinating center according to the simple urn method, ${ }^{12}$ with stratification by clinical site and gestational age category (34 to 35 weeks versus 36 weeks). Each participant's supply of study medication was packaged according to this sequence. Neither the participants nor the investigators were aware of treatment assignments. During the trial, we changed the company manufacturing the placebo and packaging the study medication. This resulted in suspension of recruitment until a new company was identified.

After administration of the study medication, the women were managed clinically according to local practice including discharge home if delivery did not occur and the patient was considered stable. For those enrolled because of an indication for preterm delivery, labor inductions were expected to start by 36 weeks 5 days, and cesarean deliveries were to be scheduled by 36 weeks 6 days and not before 24 hours after randomization. Trained and certified research staff abstracted information from maternal and neonatal charts including demographic information, medical, obstetric, and social history, and outcome data. All infants discharged on oxygen were followed up at 28 days of age to determine whether there was a continuing need for oxygen supplementation.

\section{Study Outcomes}

The primary outcome was a composite endpoint describing the need for respiratory support by 72 hours of age consisting of one or more of the following: continuous positive airway 
pressure (CPAP) or high flow nasal cannula (HFNC) for at least two consecutive hours, an oxygen requirement with a fraction of inspired oxygen $\left(\mathrm{FiO}_{2}\right)$ of at least 30 percent for at least four continuous hours, extra corporeal membrane oxygenation (ECMO) or the need for mechanical ventilation. HFNC required a flow of $>1 \mathrm{Liter} /$ minute. Stillbirth and neonatal death before 72 hours of age were also included in both composite outcomes as they are potentially competing events. Prespecified subgroup analyses for the primary outcome and severe respiratory morbidity were: $34-35$ versus 36 weeks gestation, indication for trial entry (preterm labor versus spontaneous membrane rupture versus obstetrical or medical indication), planned cesarean versus planned attempt at vaginal delivery at trial entry, infant sex, and race/ethnicity.

Neonatal secondary outcomes included: severe respiratory morbidity, a composite outcome defined as any of: CPAP or HFNC for at least 12 continuous hours, supplemental oxygen of 30 percent or more for at least at least 24 continuous hours, ECMO or mechanical ventilation, or stillbirth or neonatal death; respiratory distress syndrome (RDS); transient tachypnea of the newborn ; apnea; bronchopulmonary dysplasia (BPD); surfactant administration; need for resuscitation at birth; hypoglycemia; feeding difficulty;hypothermia,; necrotizing enterocolitis;,,intraventricular hemorrhage (IVH) Papile grade 3 or $4,{ }^{13}$; neonatal sepsis; and death before discharge. RDS was defined as the presence of clinical signs of respiratory distress (tachypnea, retractions, flaring, grunting, or cyanosis), with an oxygen requirement and a chest x-ray that showed hypoaeration and reticulogranular infiltrates. Transient tachypnea of the newborn was diagnosed when tachypnea occurred in the absence of a chest X-ray, or with a chest X-ray that was normal or showed signs of increased perihilar interstitial markings, and resolved within 72 hours. BPD was defined as a supplemental oxygen requirement $\left(\mathrm{FiO}_{2}>0.21\right)$ for the first 28 days of life. Maternal secondary outcomes included chorioamnionitis, endometritis, delivery prior to corticosteroid completion and length of stay. Definitions of all secondary outcomes are included in the Supplementary Appendix (Tables S7-S8).

Charts of all infants admitted to special care were centrally reviewed by a subgroup of the investigators and nurse coordinators to verify the respiratory outcomes. Discrepancies among reviewers, or between local research staff and the reviewers, were adjudicated by an independent neonatal consultant (LJ) who also reviewed all potential cases of BPD. All reviewers were blinded to treatment allocation.

\section{Statistical Analysis}

The expected rate of the primary outcome in the placebo group was estimated from a pilot study of infants born in the late preterm period, adjusted downward to account for the proportion of women at risk for late preterm delivery who deliver at term (20-40\%), as preterm delivery cannot reliably be predicted. We estimated that a sample size of 2,800 women would be required to detect a one third decrease in the primary outcome rate from $9.5 \%$ in the placebo arm to $6.3 \%$ in the steroid arm, with type 1 error of 5\%,2-sided, and power of at least $85 \%$. Details are in the Supplementary Appendix.

Analyses were performed according to the intention-to-treat principle. We compared continuous variables using the Wilcoxon test and categorical variables using the chi-square 
test, and Fisher's exact test. An independent data and safety monitoring committee monitored the trial. A group sequential method was used to control the Type I error rate using the Lan-DeMets characterization of the O'Brien-Fleming boundary. ${ }^{14}$ Two interim analyses were performed; in the final analysis of the primary outcome, a two-tailed $\mathrm{P}$ value of less than 0.048 was considered to indicate statistical significance. Since the adjustment is minimal we report the $95 \%$ confidence interval for the relative risk. For all secondary outcomes, a nominal $\mathrm{P}$ value of less than 0.05 was considered to indicate statistical significance, without adjustment for multiple comparisons; relative risks and $95 \%$ confidence intervals are reported. To determine whether there was a differential effect of betamethasone for the primary and severe respiratory composite outcomes within the prespecified subgroups, the Breslow-Day interaction test was conducted; where a nominal $\mathrm{P}$ value less than 0.05 was considered to indicate statistical significance.

\section{Results}

\section{Subject Characteristics}

Recruitment began in October 2010 and concluded in February 2015. Of 24,538 screened women, 2,831 eligible subjects consented and were randomized, 1,429 participants to betamethasone and 1,402 to placebo (Figure 1). The most common reason for exclusion was delivery expected within 24 hours (6,203 women; $32 \%$ ). The betamethasone and placebo groups were similar at baseline except for maternal age (mean 28.6 years versus 27.8 years, respectively, $\mathrm{P}=0.001)$ and the proportion of women of Hispanic ethnicity $(71.6 \%$ versus $67.9 \%$, respectively, $\mathrm{P}=0.03$, Table 1 ).

\section{Compliance and Side Effects}

A total of $860(60 \%)$ women in the betamethasone group and $826(59 \%)$ in the placebo group received both doses of study medication. Of those who did not receive a second dose, $1,139(95 \%)$ delivered before 24 hours. Six women did not receive any of the assigned study medication. In the placebo arm, three women consented to the trial but subsequently declined the injection, one woman delivered after randomization but before the first dose, and one received open-label betamethasone. In the betamethasone arm, one woman was in active labor with complete cervical dilation when randomized. Adverse events following injection were more common in the placebo group than betamethasone $(20.3 \%$ versus $14.1 \%, \mathrm{P}<0.001)$. Almost all $(95 \%)$ were local reactions at the injection site (details are in the Supplementary Appendix (Table S4).

\section{Neonatal Outcomes}

Four women were lost to follow-up $(0.14 \%)$; outcome information is available for 2,827 neonates. There were no stillbirths or neonatal deaths within 72 hours. The primary outcome occurred less frequently in the betamethasone group compared with the placebo group (11.6\% versus $14.4 \%$; RR $0.80,95 \%$ CI 0.66-0.97, $\mathrm{P}=0.02$, Table 2 ).. The number needed to treat to prevent one case was 35 (95\% CI 19-259). Results remained material unchanged in post-hoc analyses adjusting for maternal age and Hispanic ethnicity, and excluding infants (xx in the betamethasone group and yy in the placebo group) with congenital anomalies not recognized until after delivery. 
The severe respiratory morbidity composite outcome was also significantly reduced in the betamethasone group compared with placebo $(8.1 \%$ versus $12.1 \%$; RR $0.67,95 \% \mathrm{CI}$ $0.53-0.84, \mathrm{P}<0.001)$. The number needed to treat to prevent one case was 25 (95\% CI 16-56). The rates of RDS and apnea were similar between groups, but rates of transient tachypnea of the newborn, need for resuscitation, and BPD were significantly less frequent in the betamethasone group.(Table 2.).

None of the subgroup interaction tests for the primary outcome were significant. There was one marginally significant interaction $(\mathrm{P}=0.05)$ between treatment group and planned delivery type for the secondary severe respiratory morbidity outcome, with a significant reduction in the betamethasone group among those for whom a cesarean was planned at trial entry, but not those planning a vaginal delivery attempt. (Supplementary Appendix, Tables S9-S10).

Two infants (both in the betamethasone group) died before neonatal discharge,: one death was due to septic shock and the other to structural cardiac anomaly and arrhythmia. There were no significant differences between groups in gestational age at delivery, the frequency of small-for-gestational-age neonates, or rates of neonatal sepsis, necrotizing enterocolitis, or intraventricular hemorrhage (Table 3). As compared with Infants in the placebo group, infants in the betamethasone group were less likely to spend three or more days in the intensive or intermediate care nursery $(\mathrm{P}=0.03)$ and had a shorter time to first feed, but had a higher incidence of neonatal hypoglycemia (24.0\% vs. $14.9 \%$; RR 1.61, 95\% CI 1.38-1.99, $\mathrm{P}<0.001$ ). Additional analyses of secondary outcomes are in the Supplementary Appendix Tables S7-S8.

\section{Maternal Outcomes}

There were no significant differences between groups in the incidence of chorioamnionitis or endometritis. The rates of cesarean delivery, time to delivery, and length of stay were also similar between groups (Table 4).

Serious Adverse Events-Serious maternal adverse events occurred in 10 women in the betamethasone group and 12 in the placebo group (Supplemental appendix table xx). Serious adverse neonatal events occurred in 4 infants in the betamethasone group (in addition to the 2 deaths, one infant had reflux and one had thrombocytopenia) and 1 (supraventricular tachycardia) in the placebo group.

\section{Discussion}

This randomized multicenter trial demonstrated that antenatal administration of betamethasone to women at risk for late preterm delivery decreased the need for significant respiratory support during the first 72 hours of life. Betamethasone administration also resulted in a lower frequency of severe respiratory morbidity and BPD, less need for surfactant, and a reduction in prolonged stays in a special care nursery. Administration of betamethasone did not significantly affect peripartum maternal or neonatal infection rates, but increased the rate of neonatal hypoglycemia. 
Our findings are consistent with the results of the ASTECS trial (Antenatal Steroids for Term Caesarean Section), in which women were randomized to receive or not to receive antenatal corticosteroids at the time of elective cesarean delivery at term. There was a significant reduction in neonatal intensive care admission for respiratory morbidity in the betamethasone group (RR 0.46; 95\% CI 0.23-0.93). ${ }^{15}$ Treatment with betamethasone for patients undergoing a scheduled cesarean delivery at term has since become the standard of care in the UK.

Two smaller randomized trials have specifically assessed the use of betamethasone in the late preterm period to prevent adverse neonatal respiratory outcomes. ${ }^{16,17}$ However, these studies were inconclusive as they were underpowered, ${ }^{17}$ had substantial loss to follow-up, ${ }^{16}$ and had post-randomization exclusions. ${ }^{17}$

We found that infants exposed to antenatal betamethasone had a higher rate of neonatal hypoglycemia defined as glucose $<40 \mathrm{mg} / \mathrm{dL}$. We did not collect data on actual blood sugar concentrations. However, there were no reported adverse events related to hypoglycemia, and the rates of neonatal intensive or intermediate care admission were similar between groups. Additionally, the betamethasone group was less likely to have prolonged ( $>3 \mathrm{~d}$ ) NICU stays, suggesting the transient nature of the hypoglycemia. Few trials of antenatal corticosteroids have included information on neonatal hypoglycemia; ${ }^{4}$ however the original trial of antenatal corticosteroids found no significant difference in rates of neonatal hypoglycemia between groups. ${ }^{18}$ Nevertheless, our data support monitoring neonatal blood glucose after antenatal betamethasone exposure in the late preterm period.

Our study protocol did not allow the use of other prenatal interventions, such as tocolysis, thereby supporting that the difference in the occurrence of the primary outcome was due to antenatal corticosteroid administration. Although we delayed augmentation of labor via oxytocin by 12 hours for women with ruptured membranes who were experiencing contractions or whose cervical dilation was $3 \mathrm{~cm}$ or more, we did not find a significant difference in the rates of maternal or neonatal infectious morbidity between groups.

The challenges in predicting the timing of delivery are demonstrated by the fact that, despite inclusion and exclusion criteria designed to identify those women most likely to deliver 24 hours to 7 days after betamethasone administration, only $60 \%$ of participants received two doses of study medication. These eventualities would likely have reduced the effect of betamethasone, suggesting that the observed between group differences may underestimate effects if both doses were received.

In conclusion, the administration of antenatal betamethasone administration for women at risk for late preterm delivery significantly decreased the rate of respiratory complications in newborns. Betamethasone administration significantly increased the rate of neonatal hypoglycemia, but not other maternal or neonatal complications

\section{Supplementary Material}

Refer to Web version on PubMed Central for supplementary material. 


\section{Acknowledgments}

The authors thank Ronald Wapner, MD, and Michelle DiVito, RN, MSN, without whom this trial would not have been completed. Dr. Wapner contributed study design and protocol development, central outcome review, and expert advice. Ms. DiVito contributed administrative management and central outcome review. In addition, the authors thank Carol Blaisdell, MD (NHLBI) for advice, oversight and support; Kathleen Jablonski, PhD for study/ data management and statistical analysis; Catherine Spong, MD, (NICHD) for protocol development, central outcome review and oversight; Felecia Ortiz, RN, BSN and Sabine Bousleiman, RNC, MSN, MPH for coordination between the clinical research centers and central outcome review; Karin Kushniruk, RN, Ashley Salazar, RN, MSN, WHNP, and Mary Talucci RN for central outcome review; and Rosemary Higgins MD (NICHD) for expert advice.

Funded by the National Heart Lung and Blood Institute (grants HL098554, HL098354); by the Eunice Kennedy Shriver National Institute of Child Health and Human Development (grants HD21410, HD27915, HD27917, HD27869, HD34116, HD34208, HD40485, HD40500, HD40512, HD40544, HD40545, HD40560, HD53097, HD53118, HD68268, HD68258, HD68282, HD36801) and by the National Center for Advancing Translational Sciences, National Institutes of Health (UL1 TR000040).

\section{References}

1. Effect of corticosteroids for fetal maturation on perinatal outcomes. NIH consensus statement. 1994; 12:1-24.

2. Effect of corticosteroids for fetal maturation on perinatal outcomes. NIH Consensus Development Panel on the Effect of Corticosteroids for Fetal Maturation on Perinatal Outcomes. Jama. 1995; 273:413-8. [PubMed: 7823388]

3. Crowley PA. Antenatal corticosteroid therapy: a meta-analysis of the randomized trials, 1972 to 1994. Am J Obstet Gynecol. 1995; 173:322-35. [PubMed: 7631713]

4. Roberts D, Dalziel S. Antenatal corticosteroids for accelerating fetal lung maturation for women at risk of preterm birth. Cochrane database of systematic reviews. 2006:CD004454. [PubMed: 16856047]

5. Escobar GJ, Clark RH, Greene JD. Short-term outcomes of infants born at 35 and 36 weeks gestation: we need to ask more questions. Semin Perinatol. 2006; 30:28-33. [PubMed: 16549211]

6. ACOG technical bulletin. Preterm labor. Number 206--June 1995 (Replaces No. 133, October 1989). Int J Gynaecol Obstet. 1995; 50:303-13. [PubMed: 8543120]

7. McIntire DD, Leveno KJ. Neonatal mortality and morbidity rates in late preterm births compared with births at term. Obstet Gynecol. 2008; 111:35-41. [PubMed: 18165390]

8. Yoder BA, Gordon MC, Barth WH Jr. Late-preterm birth: does the changing obstetric paradigm alter the epidemiology of respiratory complications? Obstetrics and gynecology. 2008; 111:814-22. [PubMed: 18378739]

9. Consortium on Safe L, Hibbard JU, Wilkins I, Sun L, Gregory K, Haberman S, Hoffman M, Kominiarek MA, Reddy U, Bailit J, Branch DW, Burkman R, Gonzalez Quintero VH, Hatjis CG, Landy H, Ramirez M, VanVeldhuisen P, Troendle J, Zhang J. Respiratory morbidity in late preterm births. JAMA. 2010; 304:419-25. [PubMed: 20664042]

10. Raju TN, Higgins RD, Stark AR, Leveno KJ. Optimizing care and outcome for late-preterm (nearterm) infants: a summary of the workshop sponsored by the National Institute of Child Health and Human Development. Pediatrics. 2006; 118:1207-14. [PubMed: 16951017]

11. Martin JA, Hamilton BE, Osterman MJ, Curtin SC, Matthews TJ. Births: final data for 2013. Natl Vital Stat Rep. 2015; 64:1-65.

12. Rosenberger, W.; Lachin, JM. Randomization in Clinical Trials. Wiley; New York: 2002.

13. Papile LA, Burstein J, Burstein R, Koffler H. Incidence and evolution of subependymal and intraventricular hemorrhage: a study of infants with birth weights less than 1,500 gm. J Pediatr. 1978; 92:529-34. [PubMed: 305471]

14. Demets DL, Lan KKG. Interim Analysis - the Alpha-Spending Function-Approach. Stat Med. 1994; 13:1341-52. [PubMed: 7973215]

15. Stutchfield P, Whitaker R, Russell I. Antenatal betamethasone and incidence of neonatal respiratory distress after elective caesarean section: pragmatic randomised trial. BMJ. 2005; 331:662. [PubMed: 16115831] 
16. Porto AM, Coutinho IC, Correia JB, Amorim MM. Effectiveness of antenatal corticosteroids in reducing respiratory disorders in late preterm infants: randomised clinical trial. BMJ. 2011; 342:d1696. [PubMed: 21487057]

17. Balci O, Ozdemir S, Mahmoud AS, Acar A, Colakoglu MC. The effect of antenatal steroids on fetal lung maturation between the 34th and 36th week of pregnancy. Gynecol Obstet Invest. 2010; 70:95-9. [PubMed: 20234136]

18. Liggins GC, Howie RN. A controlled trial of antepartum glucocorticoid treatment for prevention of the respiratory distress syndrome in premature infants. Pediatrics. 1972; 50:515-25. [PubMed: 4561295]

19. Chan ISF, Zhang ZX. Test-based exact confidence intervals for the difference of two binomial proportions. Biometrics. 1999; 55:1202-9. [PubMed: 11315068] 


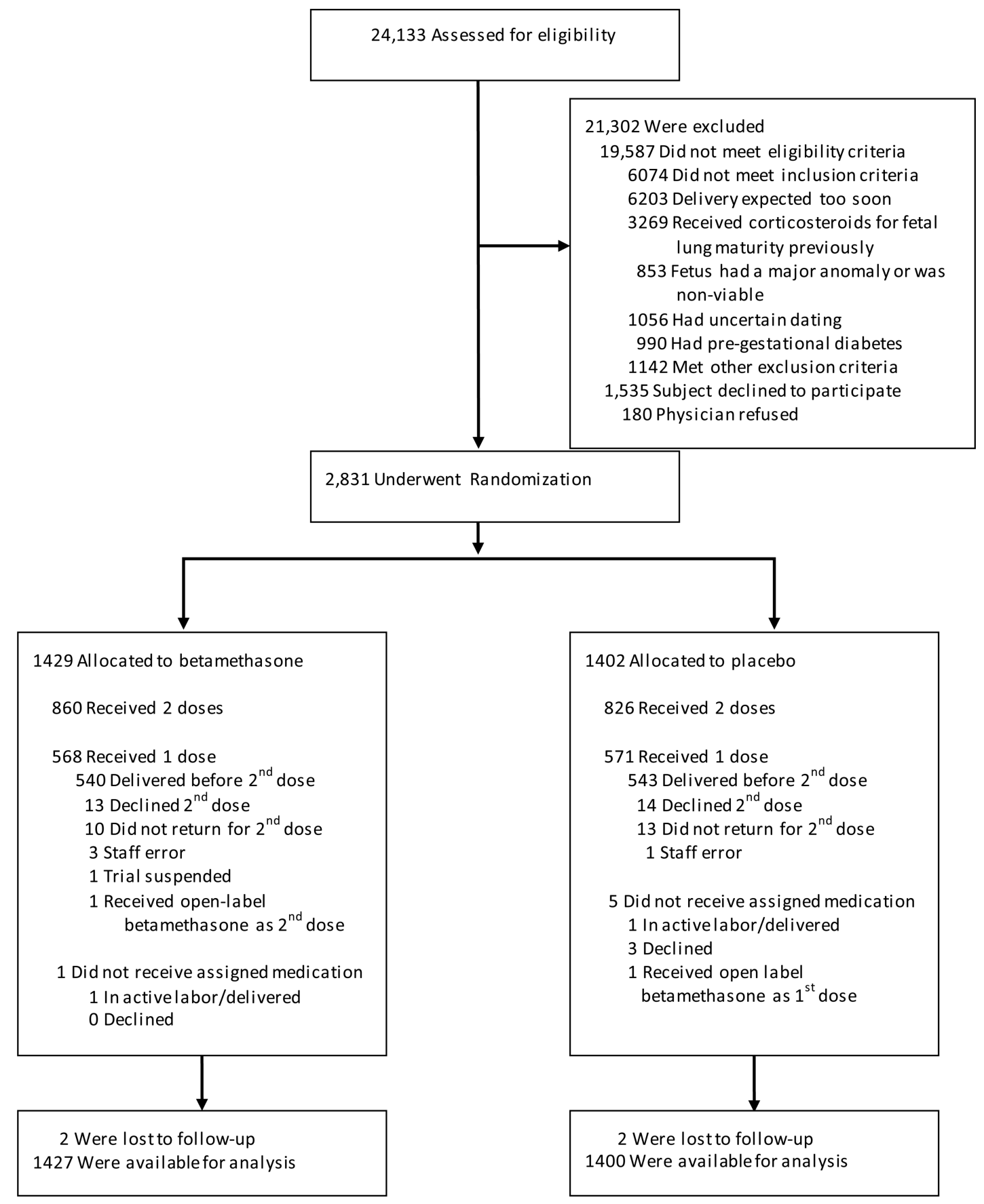

\section{Figure 1.}

Screening, Enrollment, Randomization and Follow-Up of the Study Participants 


\section{Table 1}

Baseline characteristics by treatment group

\begin{tabular}{|c|c|c|}
\hline Characteristic & Betamethasone $(\mathrm{N}=1429)$ & Placebo $(\mathrm{N}=1402)$ \\
\hline \multicolumn{3}{|l|}{ Indication for trial entry } \\
\hline Preterm labor with intact membranes & $400(28.0)$ & $392(28.0)$ \\
\hline Ruptured membranes & $316(22.1)$ & $304(21.7)$ \\
\hline Expected delivery for gestational hypertension/preeclampsia & $370(25.9)$ & $385(27.5)$ \\
\hline Expected delivery for fetal growth restriction & $46(3.2)$ & $48(3.4)$ \\
\hline Expected delivery for oligohydramnios & $50(3.5)$ & $42(3.0)$ \\
\hline Expected delivery for other indications & $247(17.3)$ & $231(16.5)$ \\
\hline \multicolumn{3}{|l|}{ Gestational age at trial entry } \\
\hline$\leq 34$ weeks & $369(25.8)$ & $399(28.5)$ \\
\hline 35 weeks & $571(40.0)$ & $532(38.0)$ \\
\hline 236 weeks & $489(34.2)$ & $471(33.6)$ \\
\hline Maternal age, years ${ }^{*}$ & $27.8 \pm 6.1$ & $28.6 \pm 6.3$ \\
\hline \multicolumn{3}{|l|}{ Race } \\
\hline Black or African American & $376(26.3)$ & $381(27.2)$ \\
\hline White & $828(57.9)$ & $800(57.1)$ \\
\hline Asian & $57(4.0)$ & $39(2.8)$ \\
\hline Other/unknown/more than one race & $168(11.8)$ & $182(13.0)$ \\
\hline \multicolumn{3}{|l|}{ Ethnicity } \\
\hline Hispanic/Latina ${ }^{* *}$ & $448(32.1)$ & $405(28.4)$ \\
\hline Not Hispanic/Latina & $949(67.9)$ & $1021(71.6)$ \\
\hline Nulliparous & $448(49.5)$ & $457(50.5)$ \\
\hline Smoking during current pregnancy & $204(14.3)$ & $186(13.3)$ \\
\hline Preeclampsia/gestational hypertension & $433(30.3)$ & $440(31.4)$ \\
\hline Gestational diabetes & $153(10.7)$ & $153(10.9)$ \\
\hline
\end{tabular}

Data expressed as $\mathrm{N}(\%)$ or mean $\pm \mathrm{SD}$

P $=0.001$

*** $\mathrm{P}=0.03$ 
Table 2

Neonatal Respiratory Outcomes

\begin{tabular}{|l|r|r|r|r|}
\hline Outcome & Betamethasone (N=1427) & Placebo (N=1400) & Relative Risk (95\% CI) & P Value \\
\hline Primary outcome & $165(11.6)$ & $202(14.4)$ & $0.80(0.66-0.97)$ & 0.02 \\
$\mathrm{CPAP}_{\text {HFNC for } \geq 2 \text { continuous hours }}$ & $145(10.2)$ & $184(13.1)$ & $0.77(0.63-0.95)$ & 0.01 \\
$\mathrm{FiO}_{2} \geq 30 \%$ for $\geq 24$ continuous hours & $48(3.4)$ & $61(4.4)$ & $0.77(0.53-1.12)$ & 0.17 \\
Mechanical ventilation & $34(2.4)$ & $43(3.1)$ & $0.78(0.50-1.21)$ & 0.26 \\
ECMO & $0(0.0)$ & $0(0.0)$ & - & N/A \\
Stillbirth or neonatal death $<72$ hours & $0(0.0)$ & $0(0.0)$ & - & N/A \\
Severe respiratory morbidity ${ }^{* *}$ & $115(8.1)$ & $169(12.1)$ & $0.67(0.53-0.84)$ & $<0.001$ \\
CPAP/HFNC for $\geq 12$ continuous hours & $93(6.5)$ & $147(10.5)$ & $0.62(0.48-0.80)$ & $<0.001$ \\
FiO 2 30\% for $\geq 24$ continuous hours & $20(1.4)$ & $34(2.4)$ & $0.58(0.33-1.00)$ & 0.05 \\
Respiratory distress syndrome & $79(5.5)$ & $89(6.4)$ & $0.87(0.65-1.17)$ & 0.36 \\
Transient tachypnea of the newborn & $95(6.7)$ & $138(9.9)$ & $0.67(0.53-0.87)$ & $<0.01$ \\
Apnea & $33(2.3)$ & $37(2.6)$ & $0.88(0.55-1.39)$ & 0.57 \\
Bronchopulmonary dysplasia & $2(0.1)$ & $9(0.6)$ & $0.22(0.02-0.92)$ & 0.04 \\
Surfactant use & $26(1.8)$ & $43(3.1)$ & $0.59(0.37-0.96)$ & 0.03 \\
Need for immediate resuscitation & $206(14.5)$ & $\mathbf{2 6 0}(\mathbf{1 8 . 7})$ & $0.78(0.66-0.92)$ & $\mathbf{0 . 0 0 3}$ \\
\hline
\end{tabular}

Data expressed as N (\%)

CPAP - continuous positive airway pressure

HFNC - high flow nasal cannula

* Primary outcome is defined as any of the following in the first 72 hours: continuous positive airway pressure (CPAP) or high flow nasal cannula (HFNC) for $\geq 2$ continuous hours; oxygen requirement with FiO2 of $\geq 30 \%$ for $\geq 4$ continuous hours; mechanical ventilation; neonatal death; or stillbirth or need for ECMO

**

Severe respiratory morbidity is defined as any of the following in the first 72 hours: CPAP or HFNC for $\geq 12$ hours; oxygen requirement with $\mathrm{FIO}_{2} \geq 30 \%$ for $\geq 24$ hours, mechanical ventilation, neonatal death; or stillbirth or need for ECMO.

${ }^{\#}$ Exact confidence limits ${ }^{19}$. 
Table 3

Other Secondary Neonatal Outcomes

\begin{tabular}{|l|r|r|r|r|}
\hline Outcome & Betamethasone N=1427 & Placebo N=1400 & RR (95\% CI) & P value \\
\hline Neonatal death & $2(0.1)$ & $0(0.0)$ & - & 0.50 \\
Birth weight - grams & $2637 \pm 480$ & $2654 \pm 484$ & & 0.32 \\
Less than $10^{\text {th }}$ percentile birth weight & $255(17.9)$ & $220(15.7)$ & $1.14(0.96-1.34)$ & 0.13 \\
Gestational age at delivery & & & & 0.10 \\
$\quad 34$ weeks & $213(15.2)$ & $193(13.5)$ & & \\
35 weeks & $386(27.6)$ & $394(27.6)$ & & \\
36 weeks & $568(40.6)$ & $609(42.7)$ & & \\
$37-38$ weeks & $185(13.2)$ & $202(14.2)$ & & \\
$39-40$ weeks & $48(3.7)$ & $29(2.0)$ & & \\
Necrotizing enterocolitis & $0(0.0)$ & $1(0.1)$ & & \\
Proven neonatal sepsis & $9(0.6)$ & $11(0.8)$ & $0.80(0.33-1.93)$ & 0.62 \\
Grade 3-4 intraventricular hemorrhage & $2(0.1)$ & $0(0.0)$ & - & \\
Hypoglycemia (glucose< 40 mg/dl) & $343(24.0)$ & $209(14.9)$ & $1.61(1.38-1.88)$ & $<0.001$ \\
Time to first feed - hours & $5.5(1.4-24.6)$ & $10.0(1.7-29.3)$ & & 0.004 \\
Intermediate nursery/NICU admission & $593(41.6)$ & $627(44.8)$ & $0.93(0.85-1.01)$ & 0.08 \\
Intermediate/NICU stay $\geq 3$ days & $470(32.9)$ & $518(37.0)$ & $0.89(0.80-0.98)$ & 0.03 \\
\hline
\end{tabular}

Data expressed as $\mathrm{N}(\%)$ or mean $\pm \mathrm{SD}$ or median $\left(25^{\mathrm{th}}-75^{\text {th }}\right.$ percentile $)$ 
Table 4

Maternal Outcomes

\begin{tabular}{l|r|r|r|r|}
\hline Outcome & Betamethasone N=1427 & Placebo N=1400 & RR (95\% CI) & P value \\
\hline Chorioamnionitis & $20(1.4)$ & $32(2.3)$ & $0.61(0.35-1.07)$ & 0.08 \\
Postpartum endometritis & $16(1.1 \%)$ & $16(1.1 \%)$ & $0.98(0.49-1.95)$ & 0.96 \\
Cesarean delivery & $454(31.8)$ & $431(30.8)$ & $1.03(0.93-1.15)$ & 0.56 \\
Randomization to delivery interval - hours & $33.0(15.2-111.6)$ & $30.6(14.6-111.0)$ & & 0.57 \\
Length of stay - days & $3(3-5)$ & $3(3-5)$ & & 0.11 \\
\hline
\end{tabular}

Data expressed as $\mathrm{N}(\%)$ or median $\left(25^{\mathrm{th}}-75^{\text {th }}\right.$ percentile $)$ 